James E. Martin

\title{
Physics for Radiation Protection
}

Third Completely Updated Edition

\section{WILEY}

WILEY-VCH Verlag GmbH \& Co. KGaA 


\section{Contents}

Preface XVII

\section{Structure of Atoms 1}

1.1 Atom Constituents 2

1.2 Structure, Identity, and Stability of Atoms 5

1.3 Chart of the Nuclides 6

$1.4 \quad$ Nuclear Models 8

Problems - Chapter 19

$2 \quad$ Atoms and Energy 11

$2.1 \quad$ Atom Measures 12

2.2 Energy Concepts for Atoms 14

2.2.1 Mass-energy 15

2.2.2 Binding Energy of Nuclei 16

2.3 Summary 18

Other Suggested Sources 18

Problems - Chapter $2 \quad 19$

3 Radioactive Transformation 21

3.1 Processes of Radioactive Transformation 21

3.1.1 Transformation of Neutron-rich Radioactive Nuclei 23

3.1.2 Double Beta $(\beta \beta)$ Transformation 27

3.1.3 Transformation of Proton-rich Nuclei 27

3.1.4 Positron Emission 29

3.1.5 Average Energy of Negatron and Positron Emitters 32

3.1.6 Electron Capture (EC) 33

3.1.7 Radioactive Transformation of Heavy Nuclei by Alpha Particle

Emission 35

3.1.8 Theory of Alpha Particle Transformation 38

3.1.9 Transuranic (TRU) Radionuclides 40

3.1.10 Gamma Emission 41

3.1.11 Internal Transition (Metastable or Isomeric States) 42

3.1.12 Internal Conversion 43 
VIII Contents

3.1.13

3.1 .14

3.1 .15

3.1 .16

3.2

3.3

3.3.1

3.3.2

3.3.3

3.3.4

3.3.5

3.3.6

3.4

3.4.1

3.5

3.5 .1

3.6

3.6.1

3.6.2

3.7

3.7.1

3.7.2

3.7 .3

3.8

3.9
Multiple Modes of Radioactive Transformation 49

Transformation by Delayed Neutron Emission 51

Transformation by Spontaneous Fission 51

Proton Emission 53

Decay Schemes 54

Rate of Radioactive Transformation 57

Activity 58

Units of Radioactive Transformation 58

Mathematics of Radioactive Transformation 60

Half-Life 62

Mean Life 63

Effective Half-life 64

Radioactivity Calculations 65

Half-life Determination 68

Activity-mass Relationships 70

Specific Activity 70

Radioactive Series Transformation 73

Series Decay Calculations 73

Recursive Kinetics: the Bateman Equations 76

Radioactive Equilibrium 77

Secular Equilibrium 78

Transient Equilibrium 80

Radionuclide Generators 81

Total Number of Transformations (Uses of $\tau$ and $\lambda_{\text {Eff) }} \quad 84$

Discovery of the Neutrino 86

Acknowledgments 87

Other Suggested Sources 87

Problems - Chapter $3 \quad 88$

$4 \quad$ Interactions 91

4.1 Production of X-rays 91

4.2 Characteristic X-rays 93 .

4.2.1 X-rays and Atomic Structure 95

4.2.2 Auger Electrons 96

4.3 Nuclear Interactions 98

4.3.1 Cross-Section 100

4.3.2 Q-values for Nuclear Reactions 102

4.4 Alpha Particle Interactions 104

4.4.1 Alpha-Neutron Reactions 105

4.5 Transmutation by Protons and Deuterons 106

4.5.1 Proton-Alpha Particle $(\mathrm{p}, a)$ Reactions 108

4.5.2 Proton-Neutron (p,n) Reactions 109

4.5.3 Proton-Gamma (p, $\gamma$ ) Reactions 110

4.5.4 Proton-Deuteron Reactions 110

4.5.5 Deuteron-Alpha (d,a) Reactions 111 
4.5.6 Deuteron-Proton (d,p) and Deuteron-Neutron $(\mathrm{d}, \mathrm{n})$ Reactions 111

4.6 Neutron Interactions 114

4.6.1 Radiative Capture $(\mathrm{n}, \gamma)$ Reactions 114

4.6.2 Charged Particle Emission (CPE) 115

4.6.3 Neutron-Proton (n,p) Reactions 116

4.6.4 Neutron-Neutron ( $n, 2 n)$ Reactions 116

4.7 Activation Product Calculations 117

4.7.1 Neutron Activation Product Calculations 119

4.7.2 Charged Particles Calculations 124

4.8 Medical Isotope Reactions 126

4.9 Transuranium Elements 128

4.10 Photon Interactions 130

4.10.1 Activation by Photons 130

4.11 Fission and Fusion Reactions 133

4.11.1 Fission 133

4.11.2 Fusion 134

4.12 Summary 138

Other Suggested Sources 139

Problems - Chapter $4 \quad 139$

$5 \quad$ Nuclear Fission and its Products 143

$5.1 \quad$ Fission Energy 145

5.2 Physics of Sustained Nuclear Fission 147

5.3 Neutron Economy and Reactivity 152

5.4 Nuclear Power Reactors 154

5.4.1 Reactor Design: Basic Systems 155

$5.5 \quad$ Light Water Reactors (LWRs) 157

5.5.1 Pressurized Water Reactor (PWR) 157

5.5.2 Boiling Water Reactor (BWR) 159

5.5.3 Inherent Safety Features of LWRs 161

5.5.4 Decay Heat in Power Reactors 163

5.5.5 Uranium Enrichment 164

5.6 Heavy Water Reactors (HWRs) 165

5.6.1 HWR Safety Systems 168

5.7 Breeder Reactors 169

5.7.1 Liquid Metal Fast Breeder Reactor (LMFBR) 171

5.8 Gas-cooled Reactors 174

5.8.1 High-temperature Gas Reactor (HTGR) 175

5.9 Reactor Radioactivity 176

5.9.1 Fuel Cladding 177

5.9.2 Radioactive Products of Fission 178

5.9.3 Production of Individual Fission Products 182

5.9.4 Fission Products in Spent Fuel 184

5.9.5 Fission Product Poisons 185

$5.10 \quad$ Radioactivity in Reactors 188 


\begin{tabular}{|c|c|c|}
\hline \multicolumn{3}{|l|}{ Contents } \\
\hline 5.10 .1 & \multicolumn{2}{|l|}{ Activation Products in Nuclear Reactors 188} \\
\hline 5.10 .2 & \multicolumn{2}{|l|}{ Tritium Production in Reactors 191} \\
\hline 5.10 .3 & \multicolumn{2}{|l|}{ Low-level Radioactive Waste 192} \\
\hline 5.11 & \multicolumn{2}{|l|}{ Summary 193} \\
\hline & \multicolumn{2}{|l|}{ Acknowledgments 194} \\
\hline & \multicolumn{2}{|l|}{ Other Suggested Sources 195} \\
\hline & \multicolumn{2}{|l|}{ Problems - Chapter 5195} \\
\hline 6 & \multicolumn{2}{|l|}{ Naturally Occurring Radiation and Radioactivity } \\
\hline 6.1 & \multicolumn{2}{|l|}{ Discovery and Interpretation 197} \\
\hline 6.2 & \multicolumn{2}{|l|}{ Background Radiation 199} \\
\hline 6.3 & \multicolumn{2}{|l|}{ Cosmic Radiation 200} \\
\hline 6.4 & \multicolumn{2}{|l|}{ Cosmogenic Radionuclides 203} \\
\hline 6.5 & \multicolumn{2}{|l|}{ Naturally Radioacitve Series 207} \\
\hline 6.5 .1 & \multicolumn{2}{|l|}{ Neptunium Series Radionuclides 214} \\
\hline 6.6 & \multicolumn{2}{|l|}{ Singly Occurring Primordial Radionuclides } \\
\hline 6.7 & \multicolumn{2}{|l|}{ Radioactive Ores and Byproducts 216} \\
\hline 6.7 .1 & \multicolumn{2}{|l|}{ Resource Recovery 218} \\
\hline 6.7 .2 & \multicolumn{2}{|l|}{ Uranium Ores 218} \\
\hline 6.7 .3 & \multicolumn{2}{|l|}{ Water Treatment Sludge 219} \\
\hline 6.7 .4 & \multicolumn{2}{|l|}{ Phosphate Industry Wastes 219} \\
\hline 6.7 .5 & \multicolumn{2}{|l|}{ Elemental Phosphorus 220} \\
\hline 6.7 .6 & \multicolumn{2}{|l|}{ Manhattan Project Wastes 221} \\
\hline 6.7 .7 & \multicolumn{2}{|l|}{ Thorium Ores 223} \\
\hline 6.8 & \multicolumn{2}{|l|}{ Radioactivity Dating 224} \\
\hline 6.8 .1 & \multicolumn{2}{|l|}{ Carbon Dating 224} \\
\hline 6.8 .2 & Dating by Primordial Radionuclides 225 & \\
\hline 6.8 .3 & Potassium-Argon Dating 226 & \\
\hline 6.8 .4 & Ionium $\left({ }^{230} \mathrm{Th}\right)$ Method 227 & \\
\hline 6.8 .5 & Lead-210 Dating 227 & \\
\hline 6.9 & Radon and its Progeny 228 & \\
\hline 6.9 .1 & Radon Subseries 229 & \\
\hline 6.9 .2 & Working Level for Radon Progeny 232 & \\
\hline 6.9 .3 & Measurement of Radon 236 & \\
\hline 6.10 & Summary 240 & \\
\hline & Acknowledgements 241 & \\
\hline & Other Suggested Sources 241 & \\
\hline & Problems - Chapter 6242 & \\
\hline 7 & Interactions of Radiation with Matter 245 & \\
\hline 7.1 & Radiation Dose and Units 245 & \\
\hline 7.1 .1 & Radiation Absorbed Dose 246 & \\
\hline 7.1 .2 & Radiation Dose Equivalent 246 & \\
\hline 7.1 .3 & Radiation Exposure 247 & \\
\hline 7.2 & Radiation Dose Calculations 249 & \\
\hline
\end{tabular}


7.2.1 Inverse Square Law 249

7.3 Interaction Processes 250

7.4 Interactions of Alpha Particles and Heavy Nuclei 252

7.4.1 Recoil Nuclei and Fission Fragments 254

7.4.2 Range of Alpha Particles 254

7.5 Beta Particle Interactions and Dose 257

7.5.1 Energy Loss by Ionization 258

7.5.2 Energy Losses by Bremsstrahlung 258

7.5.3 Cerenkov Radiation 259

7.5.4 Attenuation of Beta Particles 261

7.5.5 Range Versus Energy of Beta Particles 262

7.5.6 Radiation Dose from Beta Particles 264

7.5.7 Beta Dose from Contaminated Surfaces 267

7.5.8 Beta Contamination on Skin or Clothing 268

7.5.9 Beta Dose from Hot Particles 269

7.6 Photon Interactions 270

7.6.1 Photoelectric Interactions 271

7.6.2 Compton Interactions 272

7.6.3 Pair Production 274

7.6.4 Photodisintegration 276

7.7 Photon Attenuation and Absorption 277

7.7.1 Attenuation $(\mu)$ and Energy Absorption $\left(\mu_{\mathrm{En}}\right)$ Coefficients 280

7.7.2 Effect of $E$ and $Z$ on Photon Attenuation/Absorption 284

7.7.3 Absorption Edges 286

Checkpoints 288

7.8 Energy Transfer and Absorption by Photons 288

7.8.1 Electronic Equilibrium 293

7.8.2 Bragg-Gray Theory 295

7.9 Exposure/Dose Calculations 296

7.9.1 Point Sources 297

7.9.2 Gamma Ray Constant, $\Gamma \quad 298$

7.9.3 Exposure and Absorbed Dose 300

7.9.4 Exposure, Kerma, and Absorbed Dose 301

7.10 Summary 303

Acknowledgments 303

Other Suggested Sources 304

Problems - Chapter $7 \quad 304$

$8 \quad$ Radiation Shielding 307

8.1 Shielding of Alpha-Emitting Sources 307

8.2 Shielding of Beta-Emitting Sources 308

8.2.1 Attenuation of Beta Particles 308

8.2.2 Bremsstrahlung Effects for Beta Shielding 311

8.3 Shielding of Photon Sources 314

8.3.1 Shielding of Good Geometry Photon Sources 315 


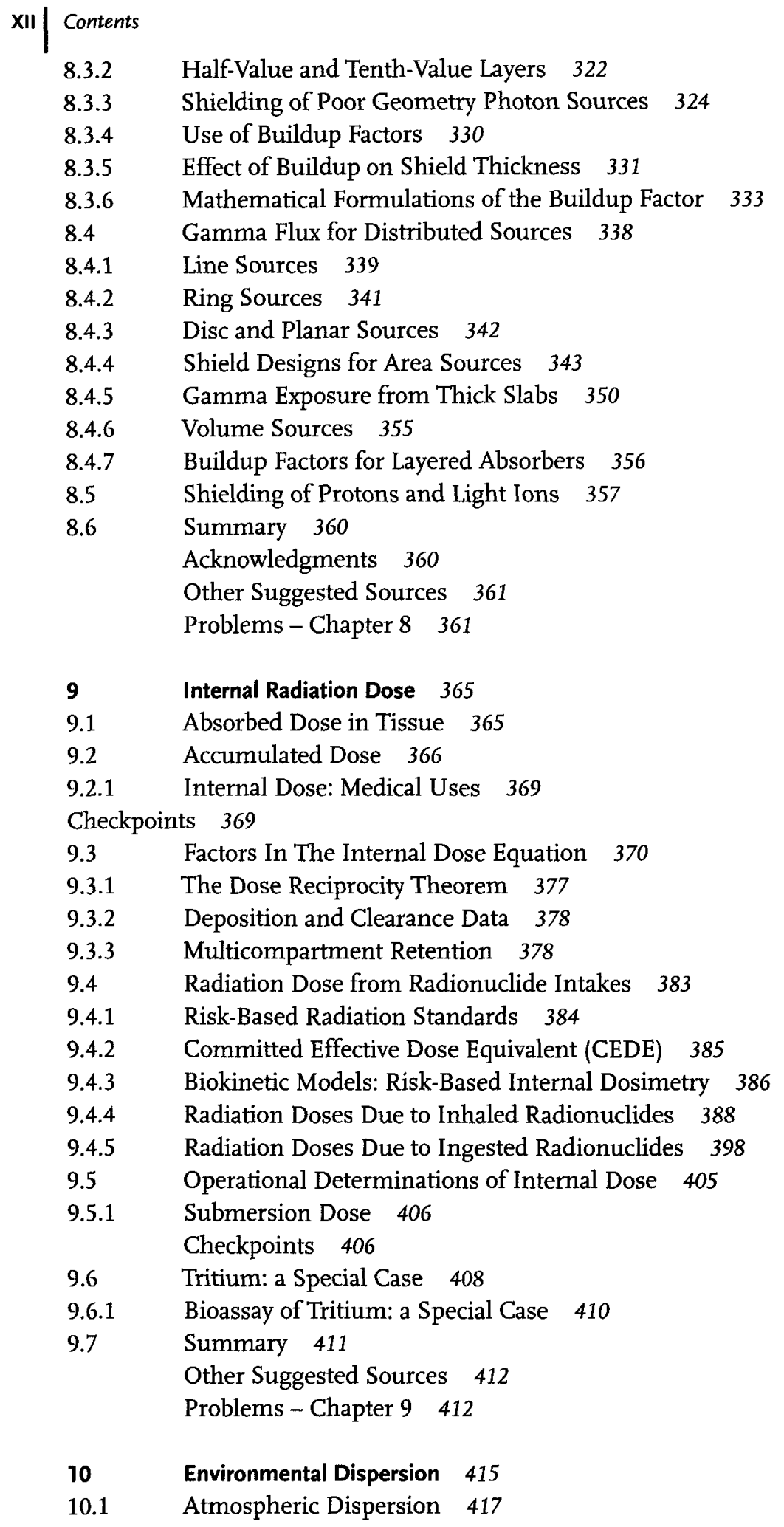


10.1.1 Atmospheric Stability Effects on Dispersion 420

10.1.2 Atmospheric Stability Classes 422

10.1.3 Calculational Procedure: Uniform Stability Conditions 424

10.1.4 Distance $x_{\max }$ of Maximum Concentration $\left(\chi_{\max }\right) \quad 426$

10.1.5 Stack Effects 427

Checkpoints 429

10.2 Nonuniform turbulence: Fumigation, Building Effects 429

10.2.1 Fumigation 429

10.2.2 Dispersion for an Elevated Receptor 431

10.2.3 Building Wake Effects: Mechanical Turbulence 432

10.2.4 Concentrations of Effluents in Building Wakes 433

10.2.5 Ground-level Area Sources 435

10.2.6 Effect of Mechanical Turbulence on Far-field Diffusion 436

10.3 Puff Releases 438

$10.4 \quad$ Sector-Averaged $\chi / Q$ Values 439

10.5 Deposition/Depletion: Guassian Plumes 443

10.5.1 Dry Deposition 443

10.5.2 Air Concentration Due to Resuspension 447

10.5.3 Wet Deposition 449

10.6 Summary 452

Other Suggested Sources 452

Problems - Chapter $10 \quad 453$

$11 \quad$ Nuclear Criticality 455

11.1 Nuclear Reactors and Criticality 456

11.1.1 Three Mile Island Accident 456

11.1.2 Chernobyl Accident 458

11.1.3 NRX Reactor: Chalk River, Ontario, December 1952461

11.1.4 SL-1 Accident 461

11.1.5 K-reactor, Savannah River Site, 1988462

11.1.6 Fukushima-Daichi Plant-Japan, March 11, 2011463

11.2 Nuclear Explosions 464

11.2.1 Fission Weapons 464

11.2.2 Fusion Weapons 465

11.2.3 Products of Nuclear Explosions 466

11.2.4 Fission Product Activity and Exposure 467

Checkpoints 469

11.3 Criticality Accidents 470

11.3.1 Y-12 Plant, Oak Ridge National Laboratory, TN: June 16, 1958470

11.3.2 Los Alamos Scientific Laboratory, NM: December 30, 1958471

11.3.3 Idaho Chemical Processing Plant: October 16, 1959, January 25, 1961, and October 17, $1978 \quad 472$

11.3.4 Hanford Recuplex Plant: April 7, 1962473

11.3.5 Wood River Junction RI: July 24, 1964473

11.3.6 UKAEA Windscale Works, UK: August 24, 1970474 


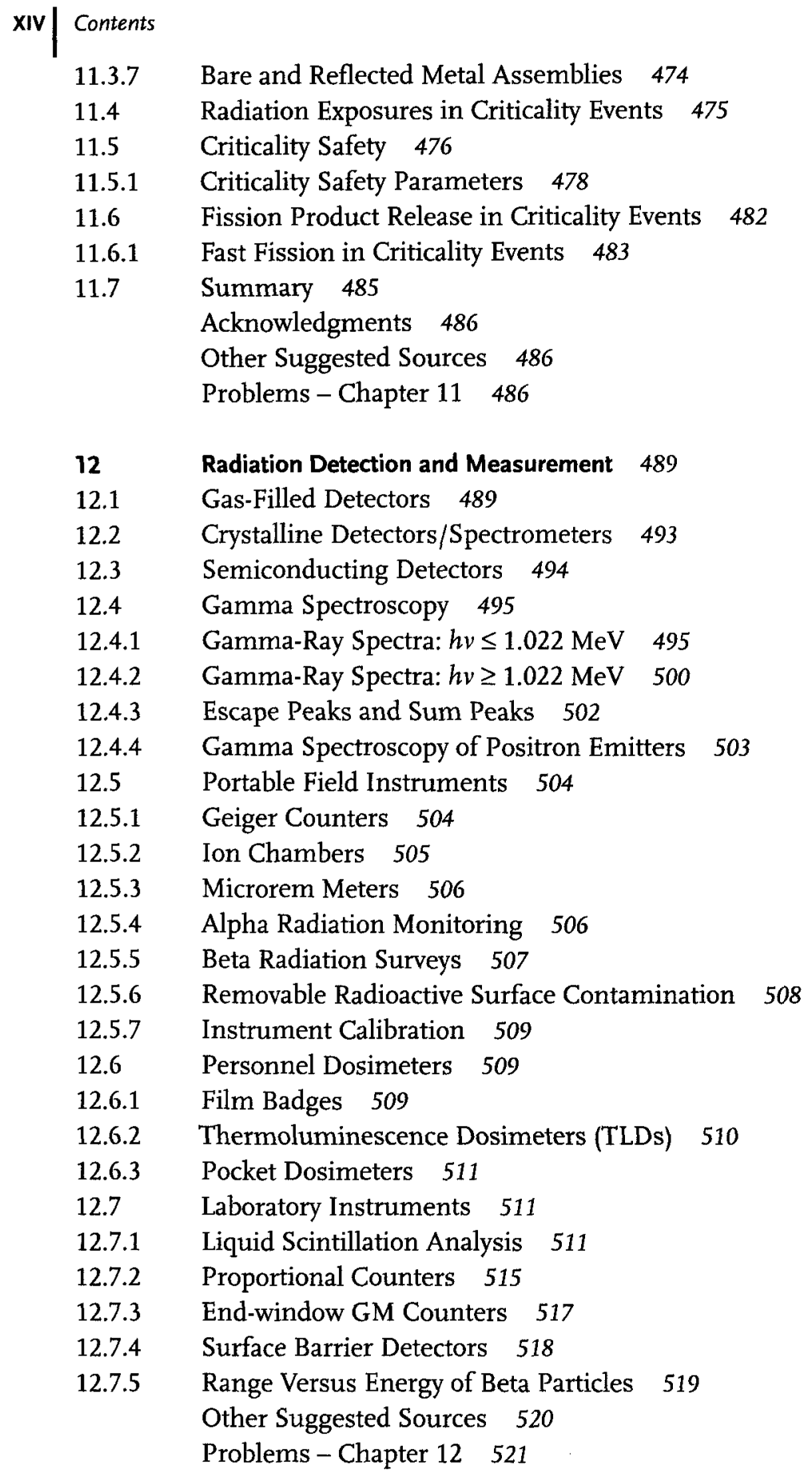


13 Statistics in Radiation Physics 523

13.1 Nature of Counting Distributions 523

13.1.1 Binomial Distribution 525

13.1.2 Poisson Distribution 525

13.1.3 Normal Distribution 527

13.1.4 Mean and Standard Deviation of a Set of Measurements 530

13.1.5 Uncertainty in the Activity of a Radioactive Source 531

13.1.6 Uncertainty in a Single Measurement 533

Checkpoints 533

13.2 Propagation of Error 534

13.2.1 Statistical Subtraction of a Background Count or Count Rate 535

13.2.2 Error Propagation of Several Uncertain Parameters 537

13.3 Comparison of Data Sets 538

13.3.1 Are Two Measurements Different? 538

13.4 Statistics for the Counting Laboratory 541

13.4.1 Uncertainty of a Radioactivity Measurement 541

13.4.2 Determining a Count Time 542

13.4.3 Efficient Distribution of Counting Time 544

13.4.4 Detection and Uncertainty for Gamma Spectroscopy 545

13.4.5 Testing the Distribution of a Series of Counts (the Chi-square Statistic) 547

13.4.6 Weighted Sample Mean 548

13.4.7 Rejection of Data 549

13.5 Levels of Detection 551

13.5.1 Critical Level 552

13.5.2 Detection Limit $\left(L_{\mathrm{d}}\right)$ or Lower Level of Detection (LLD) 554

13.6 Minimum Detectable Concentration or Contamination 558

13.6.1 Minimum Detectable Concentration (MDConc.) 558

13.6.2 Minimum Detectable Contamination (MDCont.) 560

13.6.3 Less-than Level $\left(L_{t}\right) \quad 561$

13.6.4 Interpretations and Restrictions 561

13.7 Log Normal Data Distributions 562

13.7.1 Particle Size Analysis 565

Acknowledgment 569

Other Suggested Sources 569

Chapter 13 - Problems 569

$14 \quad$ Neutrons 571

$14.1 \quad$ Neutron Sources 571

14.2 Neutron Parameters 573

14.3 Neutron Interactions 575

14.3.1 Neutron Attenuation and Absorption 576

14.4 Neutron Dosimetry 578

14.4.1 Dosimetry for Fast Neutrons 581

14.4.2 Dose from Thermal Neutrons 583 


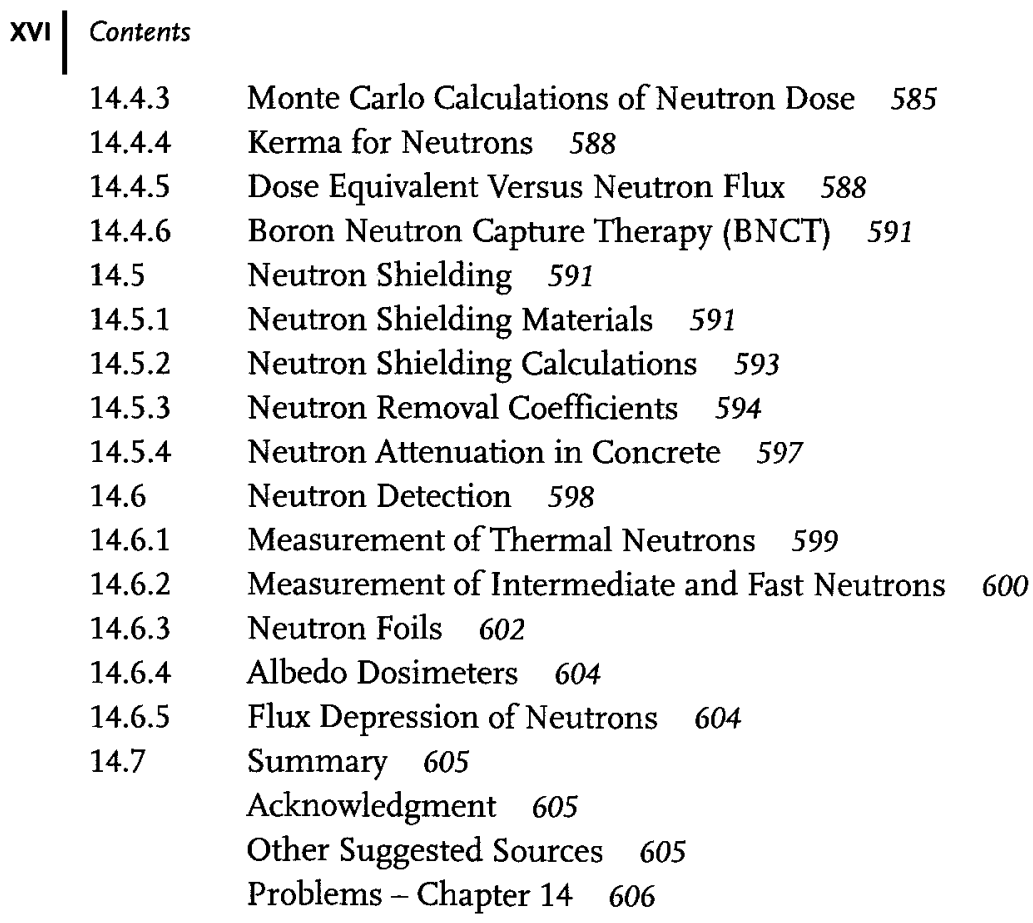

